\title{
Non-universal gravitational couplings of neutrinos in matter *
}

\author{
José F. Nieves \\ Laboratory of Theoretical Physics \\ Department of Physics, University of Puerto Rico \\ Río Piedras, Puerto Rico 00931-3343 \\ Palash B. Pal \\ Saha Institute of Nuclear Physics \\ 1/AF Bidhan-Nagar, Calcutta 700064, INDIA
}

June 14, 2021

\begin{abstract}
When neutrinos travel through a normal matter medium, the electron neutrinos couple differently to gravity compared to the other neutrinos, due to the presence of electrons in the medium and the absence of the other charged leptons. The matter-induced gravitational couplings of the neutrinos under such conditions are calculated and their contribution to the neutrino index of refraction in the presence of a gravitational potential is determined.
\end{abstract}

As is well known, the dispersion relation of a photon that propagates with momentum $\vec{K}$ in a constant gravitational potential $\phi^{e x t}$ is

$$
\omega_{K}=K\left(1+2 \phi^{e x t}\right) .
$$

This dispersion relation holds in fact not just for photons, but for any massless particle. In particular, it also holds for neutrinos, in the limit in which their mass can be neglected. This fact is a consequence of the universality of the gravitational interactions, which is embodied in the Principle of Equivalence. According to this principle, gravity couples to matter particles in a universal way; i.e., the graviton field couples to the stress-energy tensor of the particles with a coupling constant that is the same for all particle species.

*This essay received an "honorable mention" in the 1999 Essay Competition of the Gravity Research Foundation 
Since the gravitational contribution to the neutrino dispersion relation is the same for all the neutrino flavors, then it is not relevant in the context of neutrino oscillation experiments. That contribution yields a common factor in the evolution equation and therefore it drops out of the formulas for the flavor transition amplitudes. On the other hand, it has been suggested by Gasperini[1], and by Halprin and Leung[2], that a small violation of the equivalence principle, manifest as a difference in the couplings of the various neutrinos to gravity, might explain the problems associated with the observations in neutrino experiments, such as those involving solar neutrinos[3]. These authors did not attempt to specify the source of this violation of the equivalence principle, and instead assumed it as a working hypothesis. Nevertheless, by the same token, precise measurements of various observable quantities in these experiments can place significant constraints on any deviations of the Equivalence Principle in the neutrino sector.

This is an important point. Due to its prominent role in the general theory of relativity, the Equivalence Principle has been tested very accurately [4 in a variety of experiments. Those tests ordinarily involve particles that belong to the first generation of fermions, of which normal matter is made. It is much more difficult to conceive analogous tests involving the charged fermions in the other generations, since they are unstable and therefore are not amenable for performing precise tests of that kind with them. However, this may not be the case with neutrinos, which are believed to be stable particles and therefore might provide the unique opportunity for testing the equivalence principle across the generation gap, as indicated above.

In this essay we point out that a breakdown of the universality of the neutrino gravitational couplings need not involve any fundamental violation of the Equivalence Principle, but that it indeed occurs when they propagate through a medium. This violation of the Equivalence Principle does not involve any new physics beyond general relativity and the established properties of the standard particles and their interactions.

The fact that the equivalence principle is violated in an ambient thermal medium due to interactions with particles in the heat bath is known 5. Using an illustrative model of thermal QED with only photons in the background, it was demonstrated that the value of the inertial mass of a charged fermion, which is obtained from pole of its propagator, does not coincide with the value obtained from its coupling to the graviton in the weak-field limit.

Here we consider neutrinos traveling through a background of normal matter, where electrons and nucleons are present as well. By calculating the weak interaction corrections to the stress energy tensor of the neutrinos in the background, we show that the lowest order gravitational couplings of the neutrinos are modified in a non-universal way. Besides explaining the origin of the breakdown of universality in this case, the magnitude of the background-induced contribution to the neutrino gravitational couplings is calculated, the corrections to the neutrino dispersion relations in the presence of a gravitational potential 
are determined, and some of their possible phenomenological consequences are mentioned.

In the linearized theory of gravity[ [6], the graviton field is identified with the tensor $h_{\lambda \rho}$, which is defined by writing

$$
g_{\lambda \rho}=\eta_{\lambda \rho}+2 \kappa h_{\lambda \rho},
$$

where $g_{\lambda \rho}$ is the metric of the space-time and $\eta_{\lambda \rho}$ is the flat metric. The dimensional coupling $\kappa$ is related to the Newton constant by

$$
\kappa=\sqrt{8 \pi G},
$$

so that the graviton field has the properly normalized kinetic term in the linearized quantum theory of gravity. In this theory the interaction Lagrangian of the graviton field with other particles is

$$
\mathcal{L}_{h}=-\kappa h^{\lambda \rho}(x) \widehat{T}_{\lambda \rho}(x)
$$

where $\widehat{T}_{\lambda \rho}(x)$ is the stress-energy-momentum operator. This implies that a fermion-fermion-graviton vertex in a Feynman diagram is associated with the factor $-i \kappa V_{\lambda \rho}\left(p, p^{\prime}\right)$, where $p$ and $p^{\prime}$ denote the momenta of the incoming and the outgoing fermions, and

$$
V_{\lambda \rho}^{(f)}\left(p, p^{\prime}\right)=\frac{1}{4}\left[\gamma_{\lambda}\left(p+p^{\prime}\right)_{\rho}+\gamma_{\rho}\left(p+p^{\prime}\right)_{\lambda}\right]-\frac{1}{2} \eta_{\lambda \rho}\left[\left(\not p-m_{f}\right)+\left(\not p^{\prime}-m_{f}\right)\right]
$$

For the special case of chiral left-handed neutrinos,

$$
V_{\lambda \rho}^{(\nu)}\left(k, k^{\prime}\right)=\frac{1}{4}\left[\gamma_{\lambda}\left(k+k^{\prime}\right)_{\rho}+\gamma_{\rho}\left(k+k^{\prime}\right)_{\lambda}\right] L-\frac{1}{2} \eta_{\lambda \rho}\left[\not k+\not k^{\prime}\right] L
$$

where $L=\frac{1}{2}\left(1-\gamma_{5}\right)$.

When the particle propagates in a medium, this coupling is modified by interactions with the background particles. In the language of Thermal Field Theory, these modifications can be viewed as corrections to $\widehat{T}_{\lambda \rho}$ arising from loop diagrams containing thermal particles [7]. For neutrinos traveling through a normal matter medium consisting of electrons and nucleons, there are two kinds of diagrams, as shown in Figs. 1 and 2. Among them, Fig. 1 shows contributions arising from the neutral current weak interactions, which are flavor independent. On the other hand, the diagrams shown in Fig. 2 exist only for $\nu_{e}$ 's, and therefore contribute only to the $\nu_{e}$-graviton vertex without affecting the graviton vertices of $\nu_{\mu}$ or $\nu_{\tau}$. This is the source of the non-universal coupling terms.

We now turn to the calculation of the diagrams. The mass of the $W$ and the $Z$ bosons is taken to be much larger than any other mass scale in the theory and we perform the calculations to the leading order in the Fermi constant $G_{F}$. 


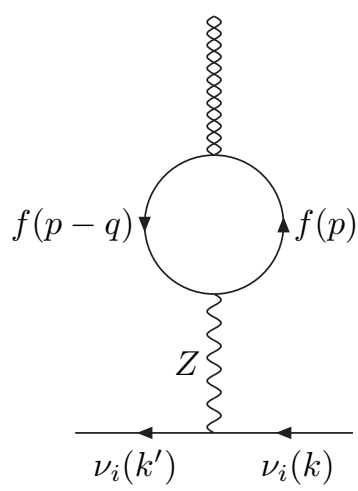

(A)

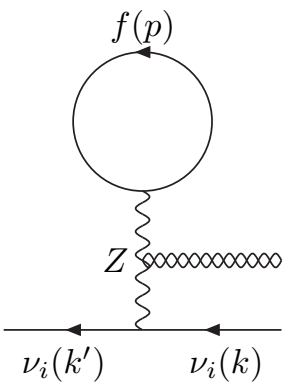

(B)

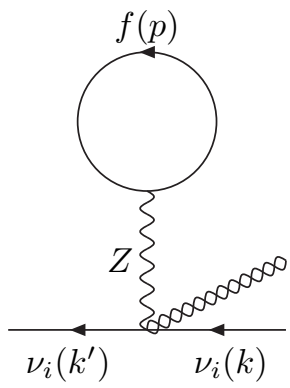

(C)

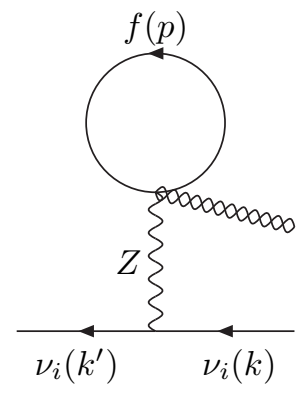

(D)

Figure 1: $Z$-exchange diagrams for the one-loop contribution to the gravitational vertex of any neutrino flavor $(i=e, \mu, \tau)$ in a background of electrons and nucleons. The braided line represents the graviton. 


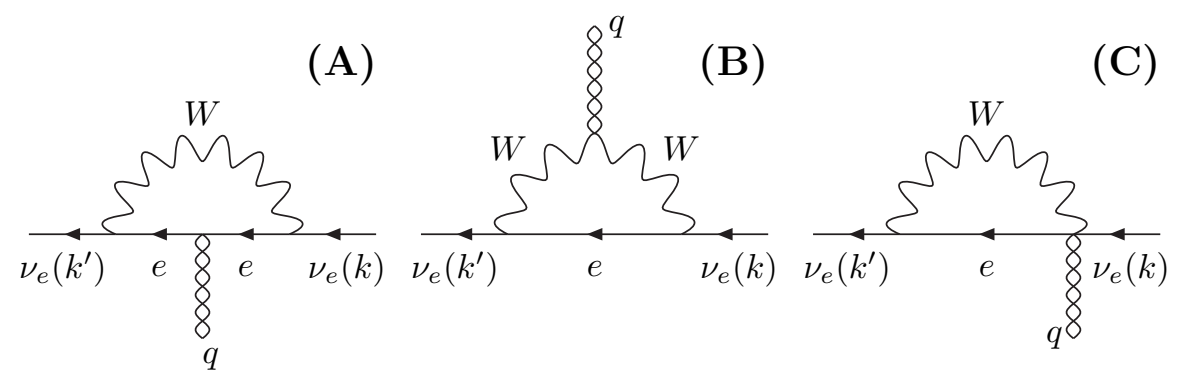

(D)

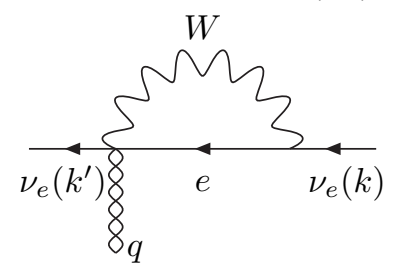

Figure 2: $W$ exchange diagrams for the one-loop contribution to the $\nu_{e}$ gravitational vertex in a background of electrons.

For the internal fermion lines we use the propagators given by the the real time formulation of Thermal Field Theory,

$$
i S_{F}^{(f)}(p)=\left(\not p+m_{f}\right)\left[\frac{i}{p^{2}-m_{f}^{2}+i \epsilon}-2 \pi \delta\left(p^{2}-m_{f}^{2}\right) \eta_{f}(p)\right],
$$

where

$$
\eta_{f}(p)=\frac{\theta(p \cdot v)}{e^{\beta\left(p \cdot v-\mu_{f}\right)}+1}+\frac{\theta(-p \cdot v)}{e^{-\beta\left(p \cdot v+\mu_{f}\right)}+1}
$$

with $\beta$ being the inverse temperature, $\mu_{f}$ the chemical potential and $v^{\mu}$ the velocity four-vector of the medium. We will work in the rest frame of the medium, where $v^{\mu}=(1, \overrightarrow{0})$.

The diagrams shown in Fig. 11A and 2A have only one $W$ or $Z$ propagator and hence give a correction of order $G_{F}$ to the gravitational vertex. Although the diagrams shown in Figs. $1 \mathrm{~B}$ and $2 \mathrm{~B}$ have two weak gauge boson propagators, the graviton coupling to the $W$ and the $Z$ also has a term which is proportional to the squared mass of the gauge boson, and so these diagrams also contribute to order $G_{F}$. These couplings can be derived by starting from the general coordinate invariant form of the pure $W$ and $Z$ Lagrangians and keeping only the 
mass terms

$$
\mathcal{L}_{g}^{(W, Z)}=\sqrt{-\mathrm{g}} g^{\lambda \rho}\left[M_{W}^{2} W_{\lambda}^{*} W_{\rho}+\frac{1}{2} M_{Z}^{2} Z_{\lambda} Z_{\rho}\right],
$$

where $\mathrm{g}=\operatorname{det}\left(g_{\lambda \rho}\right)$. To first order in $\kappa$, this yields the graviton coupling term

$$
\mathcal{L}_{h}^{(W, Z)}=\kappa h^{\lambda \rho} a_{\lambda \rho \alpha \beta}^{\prime}\left[M_{W}^{2} W^{\alpha} W^{* \beta}+\frac{1}{2} M_{Z}^{2} Z^{\alpha} Z^{\beta}\right]
$$

where

$$
a_{\lambda \rho \alpha \beta}^{\prime}=\eta_{\lambda \rho} \eta_{\alpha \beta}-\left(\eta_{\lambda \alpha} \eta_{\rho \beta}+\eta_{\lambda \beta} \eta_{\rho \alpha}\right) .
$$

In addition, there are contact couplings where the graviton couples to a vertex of non-gravitational fields. To obtain them we start with the general co-ordinate invariant form of the neutral current coupling,

$$
\mathcal{L}_{g}^{(n c)}=\sqrt{-\mathrm{g}}\left[-\frac{g}{2 \cos \theta_{W}}\left(\sum_{f} \bar{f} \gamma^{a}\left(X_{f}+Y_{f} \gamma_{5}\right) f\right)\right] v_{a}{ }^{\lambda} Z_{\lambda},
$$

where $v_{a}{ }^{\lambda}$ are the vierbeins, $X_{f}$ and $Y_{f}$ are the vector and the axial coupling with the gauge boson of the fermion $f$, and $g$ and $\theta_{W}$ are the usual parameters of the standard electroweak model. Substituting $v_{a}{ }^{\lambda}=\eta_{a}{ }^{\lambda}-\kappa h_{a}{ }^{\lambda}$, we get, to first order in $\kappa$,

$$
\mathcal{L}_{h}^{(n c)}=-\kappa \frac{g}{2 \cos \theta_{W}} h^{\lambda \rho} Z^{\beta} a_{\lambda \rho \alpha \beta} \sum_{f} \bar{f} \gamma^{\alpha}\left(X_{f}+Y_{f} \gamma_{5}\right) f,
$$

where

$$
a_{\lambda \rho \alpha \beta}=\eta_{\lambda \rho} \eta_{\alpha \beta}-\frac{1}{2}\left(\eta_{\lambda \alpha} \eta_{\rho \beta}+\eta_{\rho \alpha} \eta_{\lambda \beta}\right) .
$$

The charged current couplings with the $W$-boson can be similarly derived.

We now calculate the amplitudes corresponding to the various diagrams. The neutral current diagrams of Fig. 11 yield the following background-dependent contributions to the vertex function[8]

$$
\begin{aligned}
\Gamma_{\lambda \rho}^{(1 A)} & =\Lambda_{\lambda \rho}^{(Z)}-b^{(Z)} \psi L \eta_{\lambda \rho}, \\
\Gamma_{\lambda \rho}^{(\mathbb{B} B)} & =-b^{(Z)} a_{\lambda \rho \alpha \beta}^{\prime} \gamma^{\alpha} L v^{\beta}, \\
\Gamma_{\lambda \rho}^{(\mathbb{B} C)} & =b^{(Z)} a_{\lambda \rho \alpha \beta} \gamma^{\alpha} L v^{\beta}, \\
\Gamma_{\lambda \rho}^{(n D D)} & =b^{(Z)} a_{\rho \lambda \alpha \beta} \gamma^{\alpha} L v^{\beta} .
\end{aligned}
$$

Here, $b^{(Z)}$ is a constant whose value is not important, since the sum of the four diagrams is simply $\Lambda_{\lambda \rho}^{(Z)}$, which is given by 


$$
\begin{array}{r}
\Lambda_{\lambda \rho}^{(Z)}=\sqrt{ } 2 G_{F}\left(\gamma^{\alpha} L\right) \sum_{f} \int \frac{d^{3} p}{2 E_{f}(2 \pi)^{3}}\left\{X_{f}\left(f_{f}-f_{\bar{f}}\right)\left[\frac{N_{\lambda \rho \alpha}^{(1)}\left(p_{f}, q\right)}{q^{2}-2 p_{f} \cdot q}+(q \rightarrow-q)\right]\right. \\
\left.-Y_{f}\left(f_{f}+f_{\bar{f}}\right)\left[\frac{N_{\lambda \rho \alpha}^{(2)}\left(p_{f}, q\right)}{q^{2}-2 p_{f} \cdot q}-(q \rightarrow-q)\right]\right\},
\end{array}
$$

where $f_{f}$ and $f_{\bar{f}}$ are the momentum distribution functions for particles and antiparticles, and

$$
\begin{aligned}
N_{\lambda \rho \alpha}^{(1)}(p, q) & \equiv(2 p-q)_{\lambda}\left[2 p_{\rho} p_{\alpha}-\left(p_{\alpha} q_{\rho}+q_{\alpha} p_{\rho}\right)+(p \cdot q) \eta_{\alpha \rho}\right]+(\lambda \leftrightarrow \rho) \\
N_{\lambda \rho \alpha}^{(2)}(p, q) & \equiv(2 p-q)_{\lambda} i \epsilon_{\rho \alpha \beta \sigma} q^{\beta} p^{\sigma}+(\lambda \leftrightarrow \rho) .
\end{aligned}
$$

The same procedure for the $W$ diagrams yields

$$
\begin{aligned}
\Gamma_{\lambda \rho}^{(2 A)} & =\Lambda_{\lambda \rho}^{(W)}-b_{e} \psi L \eta_{\lambda \rho} \\
\Gamma_{\lambda \rho}^{(2 \beta)} & =b_{e}\left[-\eta_{\lambda \rho} \psi-\frac{1}{2}\left(\gamma_{\lambda} \psi \gamma_{\rho}+\gamma_{\rho} \psi \gamma_{\lambda}\right)\right] L \\
\Gamma_{\lambda \rho}^{(2) C)}=\Gamma_{\lambda \rho}^{(2 D)} & =b_{e}\left[\eta_{\lambda \rho} \psi+\frac{1}{4}\left(\gamma_{\lambda} \psi \gamma_{\rho}+\gamma_{\rho} \psi \gamma_{\lambda}\right)\right] L .
\end{aligned}
$$

Their sum is just $\Lambda_{\lambda \rho}^{(W)}$, which is obtained from Eq. (16) by making the replacements

$$
X_{e} \rightarrow 1, \quad Y_{e} \rightarrow-1
$$

and setting all other $X_{f}, Y_{f}$ to zero.

Thus, the effective gravitational vertex function for the neutrinos is

$$
\Gamma_{\lambda \rho}^{(\nu)}=V_{\lambda \rho}^{(\nu)}+\Lambda_{\lambda \rho}
$$

where

$$
\Lambda_{\lambda \rho}= \begin{cases}\Lambda_{\lambda \rho}^{(W)}+\Lambda_{\lambda \rho}^{(Z)} & \text { for } \nu_{e} \\ \Lambda_{\lambda \rho}^{(Z)} & \text { for } \nu_{\mu}, \nu_{\tau} .\end{cases}
$$

As these results clearly indicate, the effective vertex $\Lambda_{\lambda \rho}$ is not the same for all the neutrino species. As we have already argued, this is a manifestation of the fact that the background is not flavor-symmetric. One consequence of the non-universality of the induced gravitational couplings is that, in the presence of a static gravitational potential $\phi^{\text {ext }}$, the neutrino indices of refraction acquire additional contributions proportional to $\phi^{\text {ext }}$ which are flavor-dependent. To 
determine those we look at the off-shell $\nu$ - $\nu$ transition amplitude in the presence of the external potential, which is given by

$$
S_{\nu \nu}=-2 \pi i \kappa \delta\left(k^{0}-k^{\prime 0}\right)\left(V_{\lambda \rho}^{(\nu)}\left(k, k^{\prime}\right)+\Lambda_{\lambda \rho}(0, \overrightarrow{\mathcal{Q}})\right) h^{\lambda \rho}\left(\vec{k}^{\prime}-\vec{k}\right),
$$

where

$$
h^{\lambda \rho}\left(\vec{q}^{\prime}\right)=\frac{1}{\kappa} \phi\left(\vec{q}^{\prime}\right)\left(2 v^{\lambda} v^{\rho}-\eta^{\lambda \rho}\right) .
$$

In Eq. (23) $\phi\left(\vec{q}^{\prime}\right)$ stands for the Fourier transform of $\phi^{\text {ext }}$, which for a homogeneous potential is given by

$$
\phi\left(\vec{q}^{\prime}\right)=(2 \pi)^{3} \delta^{3}\left(\vec{q}^{\prime}\right) \phi^{\text {ext }} .
$$

Thus, substituting Eq. (24) into Eq. (22), we obtain the following gravitational contribution to the neutrino self-energy induced by the background

$$
\begin{aligned}
\Sigma_{G} & =\phi^{\text {ext }} V_{\lambda \rho}^{(\nu)}(k, k)\left(2 v^{\lambda} v^{\rho}-\eta^{\lambda \rho}\right)+\phi^{\operatorname{ext}} \Lambda_{\lambda \rho}(0, \overrightarrow{\mathcal{Q}} \rightarrow 0)\left(2 v^{\lambda} v^{\rho}-\eta^{\lambda \rho}\right) \\
& =\phi^{\operatorname{ext}}(\not k+2 k \cdot v \psi) L+b_{G} \psi,
\end{aligned}
$$

where

$$
b_{G}=\phi^{\operatorname{ext}} \sqrt{2} G_{F} \times \begin{cases}J_{e}+\sum_{f} X_{f} J_{f} & \text { for } \nu_{e}, \\ \sum_{f} X_{f} J_{f} & \text { for } \nu_{\mu}, \nu_{\tau},\end{cases}
$$

and

$$
J_{f}=-3\left(n_{f}-n_{\bar{f}}\right)+4 \int \frac{d^{3} p}{(2 \pi)^{3} 2 E_{f}} \frac{d}{d E_{f}}\left[\left(2 E_{f}^{2}-m_{f}^{2}\right)\left(f_{f}-f_{\bar{f}}\right)\right] .
$$

In Eq. (27) $n_{f, \bar{f}}$ denote the number density of fermions and antifermions in the background, respectively, but the evaluation of the integral can be made only if we make further assumptions about the conditions of the background material. For example,

$$
J_{f}= \begin{cases}-\beta m_{f} n_{f} & \text { classical non-relativistic gas } \\ -5 n_{f}-m_{f}^{2}\left(\frac{3 n_{f}}{\pi^{4}}\right)^{1 / 3} & \text { degenerate non-relativistic gas } \\ -5\left(n_{f}-n_{\bar{f}}\right) & \text { ultra-relativistic gas } .\end{cases}
$$

The presence of the $J_{e}$ term reflects the non-universality of neutrino couplings with the gravitational field and has the consequence that $\nu_{e}$ on one hand, and $\nu_{\mu, \tau}$ on the other, have a different gravitational contribution to their dispersion relation. If we parametrize the dispersion relation in terms of an index of refraction

$$
\mathcal{N} \equiv \frac{K}{\omega_{K}}=1-2 \phi^{\text {ext }}-\Delta \mathcal{N}_{\text {mat }}-\Delta \mathcal{N}_{G},
$$


where $\Delta \mathcal{N}_{\text {mat }}$ stands for the usual Wolfenstein term [9, 10], then Eq. (25) implies that

$$
\Delta \mathcal{N}_{G}=\frac{b_{G}}{K} .
$$

Therefore, in the presence of a static gravitational potential, the matterinduced gravitational couplings lead to new contributions to the neutrino index of refraction that could be relevant in the context of matter-enhanced neutrino oscillations. It is useful to notice that $\Delta \mathcal{N}_{\text {mat }}$ and $\Delta \mathcal{N}_{G}$ have a different dependence on the neutrino coordinate as it propagates through the medium, a property that could have distinctive implications. Whether or not these gravitational effects have observable consequences in specific contexts such as the supernova or the Solar neutrino problem, remains an open question that deserves further detailed study. However, we point out once more that the calculations that we have presented, and the results based on them, do not depend on any physical assumption beyond those required by the standard model of particle interactions and the linearized theory gravity, including the question of whether or not the neutrinos have a non-zero mass. Hence, the effects that we have considered are present at some level and it is conceivable that they are detectable in some favorable situations involving strong gravitational fields, such as those that exist in the vicinity of active galactic nuclei.

\section{References}

[1] M. Gasperini, Phys. Rev. D38, 2635 (1988);

[2] A. Halprin, C. N. Leung, Phys. Rev. Lett. 67, 1833 (1991).

[3] For a recent review and references to earlier literature see, for example, A. Dar and G. Shaviv, astro-ph/9808098.

[4] For a review of various experiments and further references see, for example, A. H. Cook, "Experiments on Gravitation", in 300 Years of Gravitation, edited by S. W. Hawking and W. Israel, Cambridge University Press 1987, pp 50 .

[5] J. F. Donoghue, B. R. Holstein, R. W. Robinett, Phys. Rev. D30, 2561 (1984) and ibid. 34, 1208 (1986).

[6] D. Boulware and S. Deser, Ann. Phys. 89, 193 (1975).

[7] J. F. Nieves and Palash B. Pal, Phys. Rev. D58, 096005 (1998).

[8] The result of the evaluation of the individual diagrams given in Ref. [7] contained some errors that originated in some incorrect signs that were adopted in the gravitational vertices involved. The results quoted below are the correct ones. However, the formulas derived there for the effective 
vertex functions $\Lambda_{\rho \lambda}^{(W, Z)}$, as well as the final results for the gravitational contribution to the neutrino self-energy and dispersion relations, coincide with those obtained here.

[9] L. Wolfenstein, Phys. Rev. D17, 2369 (1978); P. Langacker, J. P. Leveille and J. Sheiman, Phys. Rev. D27, 1228 (1983); S. P. Mikheyev and A. Yu. Smirnov, Sov. J. Nucl. Phys. 42, 913 (1985).

[10] D. Notzold and G. Raffelt, Nucl. Phys. B307, 924 (1988); P. B. Pal and T. N. Pham, Phys. Rev. D40, 259 (1989); J. F. Nieves, Phys. Rev. D40, 866 (1989); J. C. D'Olivo, J. F. Nieves and M. Torres, Phys. Rev. D46, 1172 (1992). 